Review

\title{
Metalloporphyrin Symmetry in Chiral Recognition and Enantioselective Catalysis
}

\author{
Gérard Simonneaux *, Hassan Srour ${ }^{\dagger}$, Paul Le Maux, Soizic Chevance and Daniel Carrie \\ Institute of Sciences Chimiques of Rennes, Ingénierie Chimique et Molécules pour le vivant, \\ UMR 6226 CNRS, Campus de Beaulieu, Rennes cedex 35042, France; \\ E-Mails: hassan.srour@ensicaen.fr (H.S.); paul.lemaux@univ-rennes1.fr (P.L.M.); \\ soizic.chevance@univ-rennes1.fr (S.C.); daniel.carrie@univ-rennes1.fr (D.C.) \\ $\dagger$ Present address: Laboratoire de Chimie Moléculaire et Thio-organique, University of Caen, \\ UMR 6507, Ensicaen, 6 Boulevard Maréchal Juin, Caen 14050, France. \\ * Author to whom correspondence should be addressed; E-Mail: gerard.simonneaux@univ-rennes1.fr; \\ Tel.: +33-2-2323-6285; Fax: +33-2-2323-5637.
}

Received: 28 February 2014; in revised form: 31 March 2014 / Accepted: 1 April 2014 /

Published: 10 April 2014

\begin{abstract}
Symmetry plays a fundamental role in chiral recognition and enantioselective catalysis. Porphyrins possess a number of structural features that make them attractive for the stereocontrol of chiral recognition and metal-catalyzed asymmetric reactions. This article is a brief account of our studies on chiral recognition and enantioselective catalysis by optically active metalloporphyrins. Some of the studies on chiral recognition and asymmetric catalysis by metalloporphyrins performed by others have also been included when useful.
\end{abstract}

Keywords: chiral recognition; asymmetric catalysis; asymmetric oxidation; ruthenium porphyrin; iron porphyrin; manganese porphyrin

\section{Introduction}

Symmetry and chemistry have been in a fruitful interaction in various domains of chemistry, such as catalysis, conformational analysis and chiral molecular recognition. All of these areas play an important role in biological systems. The study of the enantiomeric recognition of chiral compounds, such as amino acids, is of major concern, since these molecules are the basic building blocks of 
proteins. Natural proteins throughout evolution are made up of L-amino acids. Amino acid recognition is a key natural phenomenon, and for example, it has been recently suggested that the aminoacylation of RNA minihelices determines the homochirality of amino acids in biological systems [1]. Thus, it has often been assumed that noncovalent interactions between the side chains of aromatic or nonpolar amino acids probably play an important role in chiral recognition.

Symmetry also plays a fundamental role in enantioselective catalysis. The introduction of a symmetry element within a chiral auxiliary ligand can serve the very important function of reducing the number of possible competing diastereomeric transition states and, thus, can favor the best stereocontrol [2-4]. Biocatalysis involves the use of enzymes as catalysts for chemical reactions. Enzymes usually exhibit extremely high chemical efficiency and enantioselectivity, although the lack of rotational symmetry in the active site is generally observed. Thus, rotational symmetry is often, but not always, the key to high stereoselectivity.

The desire to mimic the functions of the hemoprotein enzymes, like cytochrome P450, led to a large development of research involving synthetic porphyrins as optically active supramolecular systems [5] and models of the active enzymatic sites [6-8]. In particular, highly convergent strategies for the synthesis of structure controlled asymmetric catalysts, using metalloporphyrins as the building blocks, are currently being developed. Catalysis by metalloporphyrins is, however, not limited to the reactions that mimic the reactions catalyzed by the cytochrome P450 family, but can be extended to different processes, which should provide original methods for asymmetric organic syntheses. Thus, the design of chiral porphyrins as asymmetric catalysts and selective receptors of various substrates is of increasing interest in the development of enantioselective catalysis and chiral recognition [9,10]. Porphyrins possess a number of structural features that make them attractive for the stereocontrol of metal-catalyzed reactions [11]. First, a number of metals can be incorporated with varying recognition sites and catalytic activities. Second, a geometrically well-defined binding pocket can be constructed consisting of a porphyrin framework and chiral recognition groups. Finally, several distinct functionalization sites, such as the meso position and the pyrrole position, can be easily used. Thus, one of the best approaches to chiral metalloporphyrin systems is to attach bulky substituents onto chiral centers near the plane of the tetrapyrrolic ring, as close as possible to the metal atom to maximize steric interaction with the incoming substrate. This article is focused on our recent work in the area of metalloporphyrin-based chiral recognition and enantioselective catalysis. Our presentation will be related directly to symmetry considerations. Some of the studies on chiral recognition and asymmetric catalysis by metalloporphyrins performed by others have also been included when useful.

\section{Results and Discussion}

\subsection{Chiral Recognition}

This part focuses on chiral recognition using ruthenium porphyrins as hosts conducted by our group. Since the pioneering work of Cram [12] and Lehn [13] on the chiral recognition of amino esters and acids by binaphthyl hosts, several approaches to the design and preparation of new chiral hosts have been reported. Many studies have been focused on metalloporphyrins as chiral hosts, because these macrocycles are quite rigid and can provide multiple chiral recognition sites by various chemical 
modifications of the porphyrin ring [10]. Our efforts in this area have been largely directed toward the systematic investigation of the reactivity of chiral ruthenium porphyrins with racemic amino acids, phosphines and isocyanides [9]. The chiral recognition of these three different ligands by optically active ruthenium porphyrins will be presented separately.

Chiral molecules containing symmetry elements are of special interest. The presence of a $\mathrm{C}_{n}$ symmetry axis within the chiral host can be very useful. We generally employed optically active carbonylruthenium(II) porphyrins as the hosts for chiral recognition in our studies. To tune their selectivity and stability, we prepared a series of $\mathrm{Ru}(\mathrm{CO})$ porphyrin hosts with different $\mathrm{C}_{n}$ symmetry (Figure 1). There has been a great deal of recent interest in ruthenium porphyrin chemistry, as viewed both in the stoichiometric and catalytic contexts. These developments seem to be parallel to the use of organometallic ruthenium catalysts containing chelating bis(tertiary phosphine) ligands for asymmetric hydrogenation [14]. Actually, ruthenium porphyrins mediate a number of interesting stoichiometric and catalytic reactions, including carbene complexation, alkane hydroxylation and alkene epoxidation. The interest in ruthenium chemistry was inspired by the periodic relationship of ruthenium to iron and the possibility of preparing relatively stable oxoruthenium derivatives [15].

\subsubsection{Chiral Recognition of Amino Acid Derivatives}

In an effort to obtain a chiral ruthenium host with a $\mathrm{C}_{2}$ axis, we first turned our attention to the preparation of ruthenium's picket-fence porphyrins bearing optically active $\alpha$ (methoxy- $\alpha$-(trifluoromethyl)phenylacetyl residues on both sides of the porphyrin plane [16] (Compound 1, Figure 1). For various amino esters, chiral recognition was observed for the complexation of ligands with up to $52 \%$ enantiomeric excess for tert-leucine methyl ester (Figure 2). The chiral recognition observed on the complexation of amino esters, which is, at first, surprising, given the lack of enantioselectivity exhibited in the synthesis of the Complex (1) Ru(valine methyl ester) ${ }_{2}$ from the bis(acetonitrile) species [17], prompted us to do some additional studies. One explanation for the chiral recognition could be the kinetics of the axial ligand rate dissociation: no ligand exchange was observed in the bis(amino ester) derivative. In contrast, the exchange of pure (L)-valine methyl ester ruthenium carbonyl Complex 1 with pure (D)-valine methyl ester at room temperature in dichloromethane (12 equivalents) leads to the formation of the other isomer with a $78 \%$ yield after a few minutes. For this reason, the dissociation rate constants of the two enantiomers of valine methyl ester were determined by ${ }^{1} \mathrm{H}$ NMR using magnetization transfer experiments, showing that the origin of the enantioselectivity in favor of the (L)-valine ligand ( $c$ a. 2.6:1) resides in the difference between the kinetics of the axial ligand dissociation of the two enantiomers. In the presence of an excess of ligand, the ligand exchange proceeds by a dissociative mechanism, with a five-coordinate transition state.

In an attempt to obtain further information with regards to the existence of noncovalent interactions between amino acid side chains and the porphyrin ligand, we prepared a series of amine and amino ester complexes with $\mathrm{C}_{4}$-symmetric ruthenium(II) porphyrins [18]. Ruthenium(II) porphyrins form stable complexes with amine ligands [19]. The choice of the Halterman porphyrin [20] (Figure 1, Compound 2) was dictated by two criteria. First, the chiral porphyrin is a rigid ligand with $\mathrm{D}_{4}$ symmetry. Second, the substituents on the arenes are aliphatic without any functional groups. The chiral recognition is directly related to the steric difference between the sterically demanding ethano 
bridge and the small methano bridge of the norbornane moiety. The addition of amines and amino esters to the ruthenium Halterman porphyrin complex immediately gave the mixed ligated species with ligation of the amino ligand in the trans position to carbon monoxide (Figure 2). For all ligands, except the phenylalanine methyl ester, the (S)-enantiomer was preferably ligated. The chiral recognition varied from $13 \%$ to $60 \%$ of enantiomeric excess (ee) [18].

Figure 1. The structure of optically active metalloporphyrin compounds.

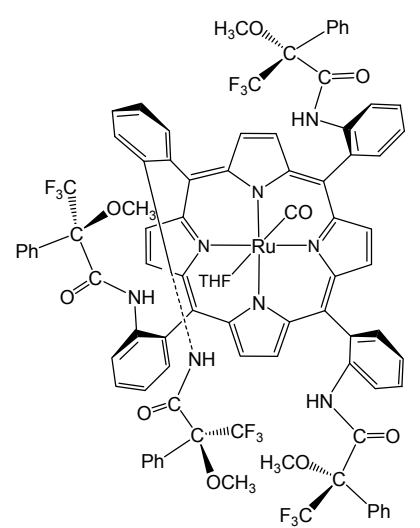

1

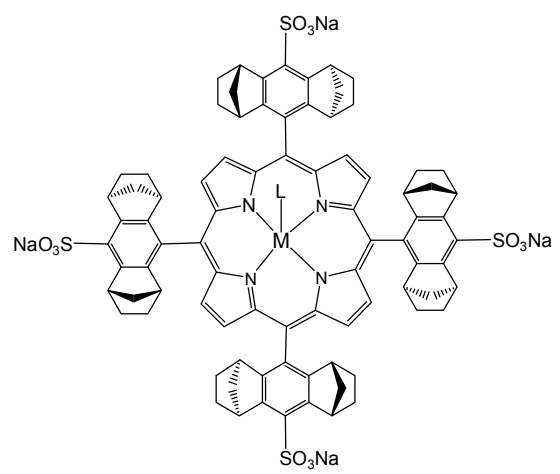

3a : M-L = Ru-CO

3b : $\mathrm{M}-\mathrm{L}=\mathrm{Fe}-\mathrm{Cl}$

3c : $\mathrm{M}-\mathrm{L}=\mathrm{Mn}-\mathrm{Cl}$

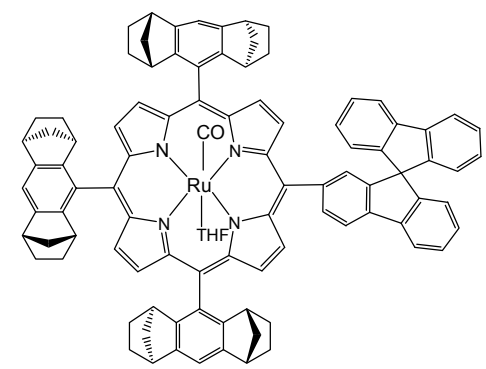

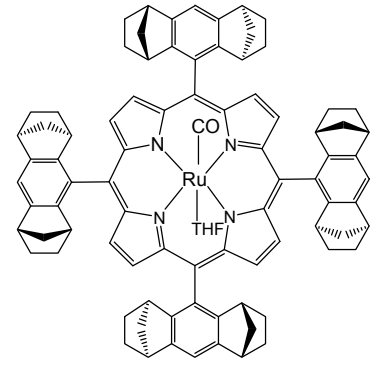

2

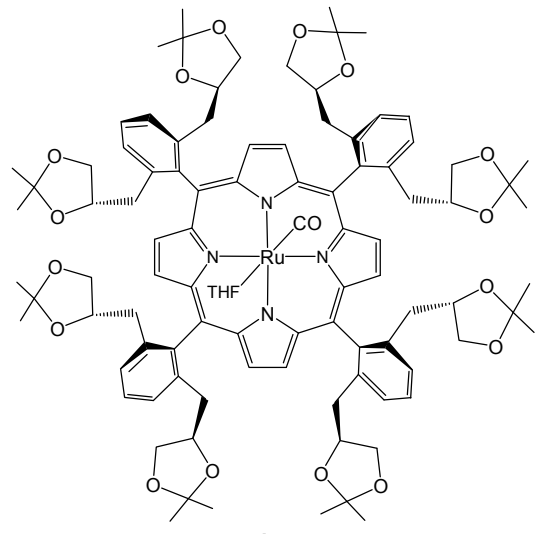

4

5

The introduction of polar functional groups into the porphyrin was used to modify the solubility, as shown for the sulfonated porphyrins, with improved solubility in water. Thus water-soluble ruthenium porphyrins with $\mathrm{C}_{4}$ symmetry were also prepared in our group from Halterman porphyrin (Figure 1, Compound 3a). This allows us to compare the chiral recognition in solvent with different polarities, and it should be noted that there are very few chiral recognitions by porphyrins in aqueous solution [21]. 
Figure 2. Complexation of amino esters to chiral ruthenium compounds.
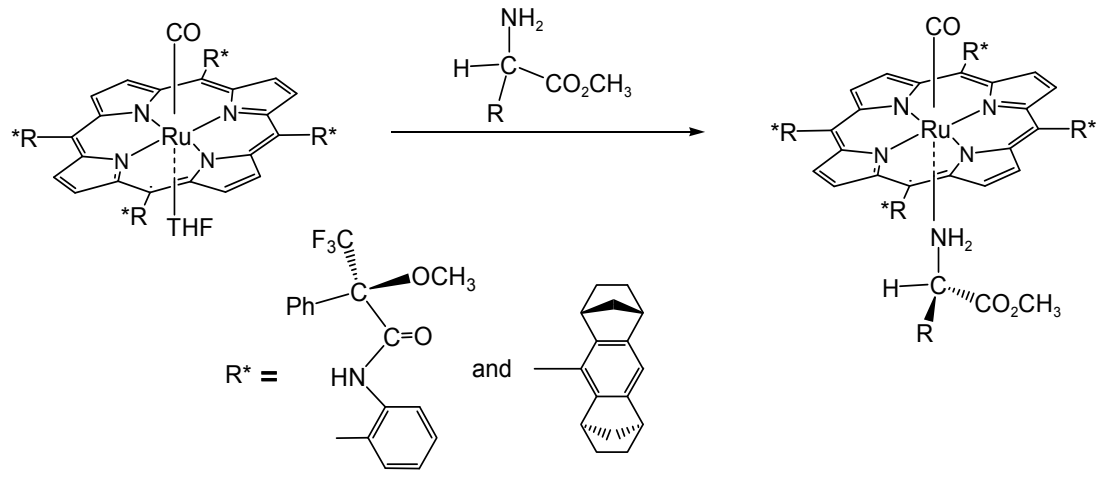

To mimic in vivo chiral recognition, water-soluble receptors that are able to show high affinity towards the targeted substrate and overcome the strong competition of water molecule were developed [5,22]. The study of the recognition process in aqueous solutions can help to reveal the nature of the binding forces involved in the chiral recognition. For this reason, it seems desirable to compare the chiral recognition observed in chloroform (with amino esters) using $\mathbf{2}$ as a host, with the chiral recognition observed in water (with amino acids) using $\mathbf{3 a}$ as a water-soluble host (Figure 1). First, it is worth noting that the recognition ability for amino acids in water is comparable, although to a lesser extent, to that of amino esters in chloroform, since the same configuration was preferred. The decrease was from $60 \%$ ee in chloroform to $28 \%$ ee in water for phenylalanine derivatives. Accordingly, this result supports the suggestion that hydrophobic interaction will not be a dominant factor (at least favorable) in chiral recognition by ruthenium porphyrins. A similar conclusion was proposed by Imai et al. [21] for the chiral recognition of amino acids by zinc porphyrins in water. As these authors suggested, the chiral recognition in aqueous solution was realized based on weak interactions other than hydrogen bonds.

\subsubsection{Chiral Recognition of Phosphines with Ruthenium Porphyrins}

To achieve chiral recognition, the chiral groups should also be inert to oxidation and to racemization. To design a metalloporphyrin, which satisfies the requirement above, we utilized a porphyrin inert framework, using the Mosher reagent to provide chiral groups and a ruthenium coordination site [23]. In order to overcome the severe problems that attend the resolution and assignment of ${ }^{1} \mathrm{H}-\mathrm{NMR}$ signals from complex materials, such as chiral porphyrins, the presence of a NMR probe in the system is needed. Of particular interest is the judicious introduction of fluorine into the chiral pickets. Thus, fluorine signals will be easier to detect than proton resonances, and because the fluorines are different for the four atropisomers, the identification of each isomer will be facilitated. For the purpose of chiral recognition, it was decided that the $\alpha, \beta, \alpha, \beta$ isomer offered the greater simplicity, because the chiral pickets of this atropisomer can provide a ruthenium porphyrin with two topologically identical faces. Thus, we used [23] the optically active ruthenium porphyrins $\left(\mathrm{C}_{2}\right.$ symmetry), derived from the addition of the Mosher reagent to tetra(aminophenyl)porphyrins (Figure 1, Compound 1). Complexation of racemic benzylmethylphenylphosphine to the $\alpha, \beta, \alpha, \beta$ isomer leads to the formation of one of three possible product diastereoisomers (bis-phosphine complexation) with high a degree of selectivity (Figure 3a). This result was obtained under kinetic control, whereas thermodynamic control yielded less favorable recognition [23]. 
Figure 3. (a) Complexation of chiral phosphine to ruthenium porphyrins; (b) complexation of chiral isocyanides to ruthenium porphyrins.
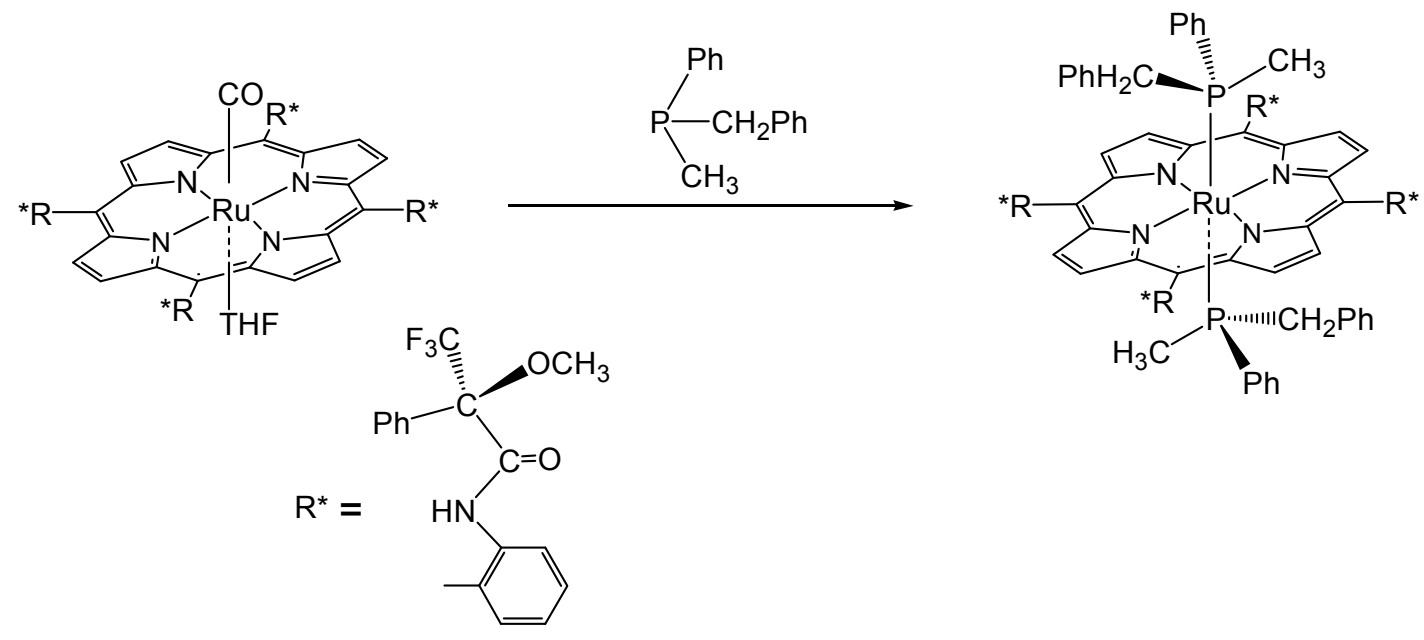

(a)
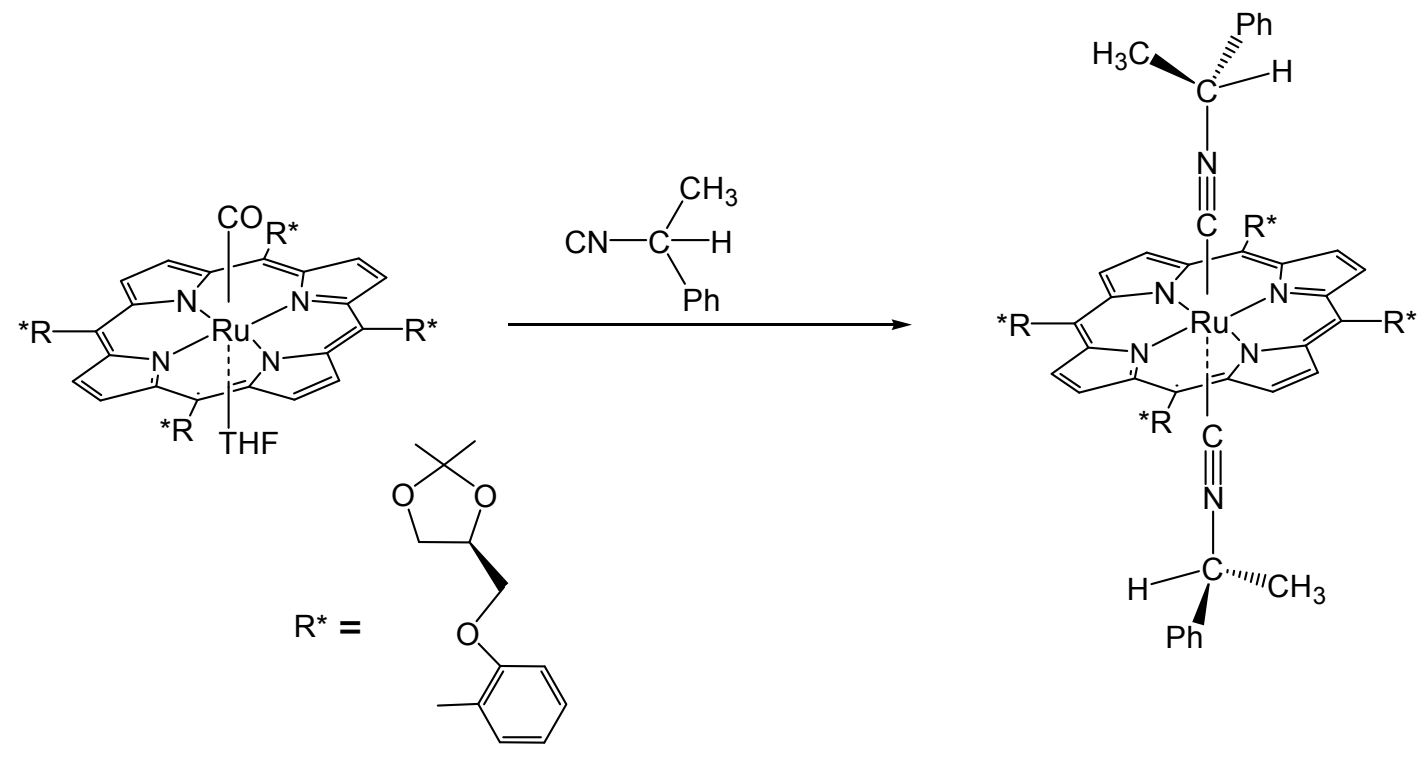

(b)

\subsubsection{Chiral Recognition of Isocyanides with Ruthenium Porphyrins}

As part of our continuing effort to develop chiral ruthenium porphyrins [9], we also reported [24] a new chiral system containing eight $D-\alpha-\beta$-isopropylideneglycerol residues linked to the ortho meso-phenyl positions via ether bonds (Figure 1, Compound 4), as well as its use for the chiral recognition of isocyanides. To be effective, the chirality centers of metalloporphyrins need to be close to the coordination center. To build such a cavity, chiral entities should be either linked directly on the meso position of the ring or linked on the ortho phenyl positions of the porphyrin ring. For the purpose of molecular recognition, it was decided that the later possibility offered the greater synthetic simplicity. Thus, we used 5,10,15,20-tetrakis(2,6-dihydroxyphenyl)porphyrin as a precursor. It is easily available from the commercial 2,6-dimethoxybenzaldehyde and was successfully used by Gross [25] to prepare a homochiral macrocycle. As expected for a $\mathrm{D}_{4}$ symmetry, all the pickets are equivalent in ${ }^{1} \mathrm{H}$ NMR spectroscopy, and thus, only one singlet is observed for the pyrrole protons. After ruthenium insertion, 
chiral recognition in the complexation of racemic isocyanides to the metal (Figure $3 \mathrm{~b}$ ) leads to the formation of three isomers with low enantioselectivity (15\%). The long distance between the ruthenium center and the chiral ligand may explain this week selectivity.

In summary, this part shows our recent progress in this field with a particular emphasis on the advantages of a supramolecular approach to chirality recognition and detection.

\subsection{Enantioselective Catalysis}

In principle, chiral catalysis is the best method to introduce stereochemistry into pharmaceutically active molecules. This is reflected by the fact that papers and reviews have appeared at an ever increasing rate since the end of the 1960 s, when the first papers using chiral metal catalysts were published by Knowles [26] and Horner [27]. Since then, the discoveries made by Noyori and Sharpless [28] have had a very great impact on academic research and the development of new drugs and materials, and they are used in many industrial syntheses of drugs and other biologically active compounds. This second part focuses on asymmetric catalysis using metalloporphyrins (metals: Fe, $\mathrm{Ru}, \mathrm{Mn}$ ) as catalysts conducted by our group. There are excellent and recent reviews devoted to this topic $[29,30]$.

\subsubsection{Asymmetric Cyclopropanation Reaction}

As part of our continuing effort to promote ruthenium porphyrin chemistry, we present now the use of the chiral porphyrin complex as a catalyst for asymmetric cyclopropanation of styrene derivatives. Enantioselective carbene transfer to olefins is an important area of asymmetric synthesis [31]. Recent growth in the area of transition metal porphyrin chemistry has, in part, been driven by the increased interest associated with metal-catalyzed cyclopropanation. Despite the periodic relationship of ruthenium to iron and osmium and the syntheses of different carbene complexes of ruthenium porphyrins, elegantly developed by Collman et al. [32], it is only in 1997 that cyclopropanation reactions were observed using ruthenium porphyrins as catalysts (Figure 4) [33].

The reaction of styrene with ethyl diazoacetate in the presence of the dioxoruthenium(VI) picket-fence complex bearing optically active $\alpha$-methoxy- $\alpha$ (trifluoromethyl) phenylacetyl residues on both sides of the porphyrin plane $\left(\alpha, \beta, \alpha, \beta\right.$ isomer, $\mathrm{D}_{2}$ symmetry) gave a mixture (trans/cis: $\left.9 / 1\right)$ of optically active cyclopropane derivatives, i.e., trans- and cis-2-phenylcyclopropanecarboxylic esters with $14 \%$ and $34 \%$ ee, respectively [33]. Better enantioselectivity was obtained with a chiral ruthenium porphyrin showing higher symmetry. Thus, we also described a general method for the highly enantioselective synthesis of cyclopropylphosphonates using $\mathrm{D}_{4}$-symmetric ruthenium porphyrin (Figure 4b) [34]. We also presented the synthesis of a series of chiral ruthenium porphyrins wherein each porphyrin bears, at least, a chiral group (containing norbornane groups fused to the central benzene ring) and a spirobifluorenyl group for future polymerization (Figure 1, Compound 5). After ruthenium insertion, all of the chiral complexes were tested for asymmetric cyclopropanation of styrenes, showing the increasing effect of the number of chiral sites on the ee. The reaction proceeded under mild conditions and gave trans-cyclopropylphosphonates with good yields and high ee's (up to 92\%). Surprisingly, Compound 5 (Figure 1) bearing only three chiral groups catalyzed the cyclopropanation reaction, yielding cyclopropylphosphonates with a moderate decrease of the ee by 
comparison with those obtained with a complex with $\mathrm{D}_{4}$ symmetry. For example, the ee decreased from $90 \%$ to $77 \%$ for styrene at $25{ }^{\circ} \mathrm{C}$. Thus, the stereochemical effect of the loss of the $\mathrm{D}_{4}$ symmetry was not so dramatic. Furthermore, replacing two chiral groups by two spirobifluorene groups yields the complex showing $\mathrm{D}_{2}$ symmetry, which catalyzed the cyclopropanation of styrene with $66 \%$ ee. Similar effects were observed using ethyl diazoacetate instead of diazomethylphosphonate. A progressive increase for stereochemical effectiveness exists between enantiomeric excess and the number of chiral groups linked to ruthenium porphyrins.

Figure 4. (a) Cyclopropanation of styrene with ethyl diazoacetate; (b) cyclopropanation of styrene with diisopropyl diazomethylphosphonate.

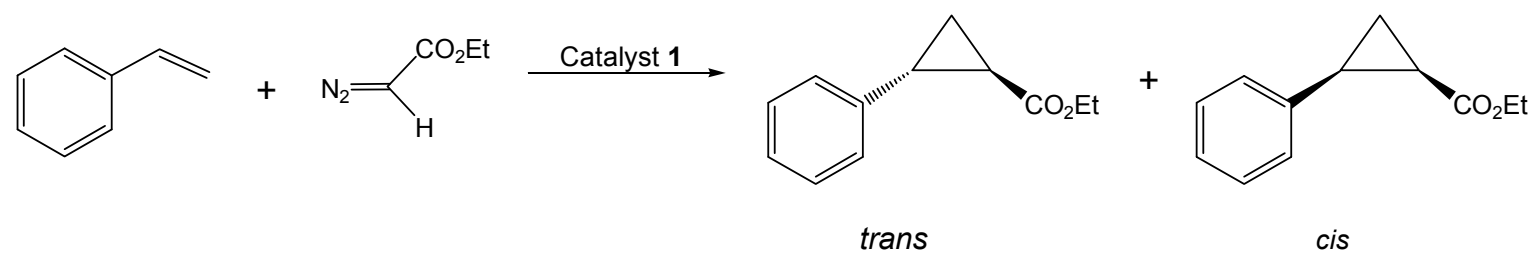

(a)

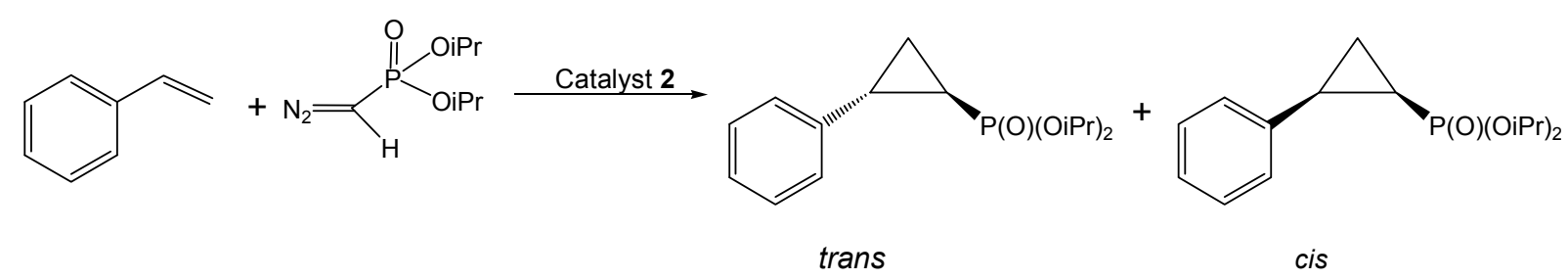

(b)

Berkessel et al. [35] and Che et al. [36] also reported independently that the ruthenium complex of the same chiral porphyrin, previously reported by Halterman, can be used to catalyze the cyclopropanation of styrene with ethyl diazoacetate. This reaction is particularly interesting, since the enantiomeric excesses are quite high (90\%). Later, Gross et al. described the asymmetric cyclopropanation of styrene by an enantiopure carbenoid under catalysis by ruthenium porphyrins [37].

\subsubsection{Asymmetric Oxidation Reactions}

Catalytic asymmetric epoxidation reactions play a major role in organic chemistry, since the optically active epoxides are important building blocks [38,39]. In particular, catalytic asymmetric reactions in aqueous solutions are attractive, but rare. Therefore, until 2006, the results obtained in the asymmetric epoxidation with $\mathrm{H}_{2} \mathrm{O}_{2}$ and chiral metal catalysts have been rather disappointing [40]. The main obstacle when using $\mathrm{H}_{2} \mathrm{O}_{2}$ is the high activity of transition-metal complexes in the catalase reaction and the homolytic cleavage of the peroxidic $\mathrm{O}-\mathrm{O}$ bond with the formation of the hydroxyl radical [6].

Our group reported the asymmetric oxidation of sulfides into sulfoxides using macroporous resins containing chiral metalloporphyrins [41] and, more recently, catalytic sulfoxidation reactions with $\mathrm{H}_{2} \mathrm{O}_{2}$ in water using a chiral iron porphyrin as a catalyst (Figure 5) [42]. We also described recent chiral epoxidation of alkenes in water/methanol solutions using $\mathrm{H}_{2} \mathrm{O}_{2}$ as an oxidant catalyzed by new optically active water-soluble iron [43] and manganese porphyrins [44] (Figure 5). A chiral water-soluble Halterman porphyrin, due to the presence of four sulfonate groups at the para-position, was previously reported by 
our group and used for the chiral recognition of amino acids [18] and catalytic carbene transfer in water [45]. The starting point of the work described here was the introduction of four sulfonate groups into an optically active porphyrin with the aim of preparing water soluble porphyrins. We choose a $\mathrm{C}_{2}$-symmetric group, which contains two norbornane groups fused to the central benzene ring, previously reported by Halterman and Jan [46]. The resulting metalloporphyrin after manganese insertion (Figure 1, Compound 3c) is an electron-rich manganese porphyrin, which may tend to favor the $\mathrm{O}-\mathrm{O}$ hemolysis of hydroperoxide. The asymmetric epoxidation of alkene and hydroxylation of arylalkane derivatives by $\mathrm{H}_{2} \mathrm{O}_{2}$ to give optically active epoxides (ee up to 68\%) and alcohols (ee up to $57 \%$ ), respectively, were carried out in water/methanol solutions using these chiral water-soluble manganese porphyrins as catalysts. Ongoing work includes investigations of an extended range of substrates, particularly those of pharmaceutical importance, further optimization of the reaction medium and more experiments to identify the precise role of chirality in the mechanism.

Figure 5. Oxidation of sulfides, alkenes and ethylbenzene by hydrogen peroxide catalyzed by metalloporphyrins (Fe, Mn).

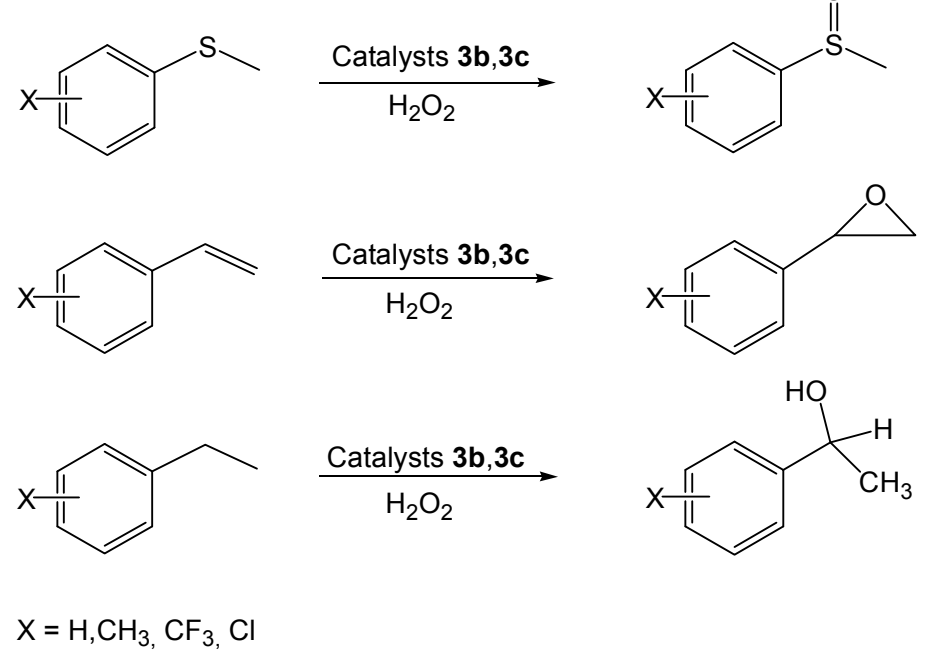

\section{Conclusions}

The work described in this review confirms the observation that chiral metalloporphyrins are particularly well adapted as asymmetric devices both for chiral recognition and catalysts for asymmetric syntheses. Our efforts in this domain will continue as we seek to design highly convergent strategies for the synthesis of symmetry-controlled novel asymmetric catalysts, using various metalloporphyrins as building blocks. Thus, chiral metal-organic frameworks might be attractive targets as catalyst supports for heterogeneous catalysis in the near future [47].

\section{Author Contributions}

Part of the experimental work on catalysis was carried out by Hassan Srour and Paul Le Maux. The experimental studies on chiral recognition were carried out by Soizic Chevance and Daniel Carrie. Gerard Simonneaux conceived the research, wrote the paper and approved the final manuscript. 


\section{Conflicts of Interest}

The authors declare no conflict of interest.

\section{References}

1. Tamura, K.; Schimmel, P.R. Chiral-selective aminoacylation of an RNA minihelix: Mechanistic features and chiral suppression. Proc. Natl. Acad. Sci. USA 2006, 103, 13750-13752.

2. Whitesell, J.K. C 2 Symmetry and asymmetric induction. Chem. Rev. 1989, 89, 1581-1590.

3. Pfaltz, A. Chiral semicorrins and related nitrogen heterocycles as ligands in asymmetric catalysis. Acc. Chem. Res. 1993, 26, 339-345.

4. Moberg, C. $\mathrm{C}_{3}$ Symmetry in asymmetric catalysis and chiral recognition. Angew. Chem. Int. Ed. Engl. 1998, 37, 248-268.

5. Borovkov, V.V.; Mamardashvili, N.Z.; Inoue, Y. Optically active supramolecular systems based on porphyrins. Russ. Chem. Rev. 2006, 75, 737-748.

6. Meunier, B. Metalloporphyrins as versatile catalysts for oxidation reactions and oxidative DNA cleavage. Chem. Rev. 1992, 92, 1411-1456.

7. Simonneaux, G.; Tagliatesta, P. Metalloporphyrin catalysts for organic synthesis. J. Porphyr. Phtalocyanins 2004, 8, 1166-1171.

8. Mansuy, D. A brief history of the contribution of metalloporphyrin models to cytochrome P450 chemistry and oxidation catalysis. C. R. Chim. 2007, 10, 392-413.

9. Simonneaux, G.; Le Maux, P. Optically active ruthenium porphyrins: Chiral recognition and asymmetric catalysis. Coord. Chem. Rev. 2002, 228, 43-60.

10. Hembury, G.A.; Borovkov, V.V.; Inoue, Y. Chirality-sensing supramolecular systems. Chem. Rev. 2008, 108, 1-73.

11. Ogoshi, H.; Mizutani, T. Multifunctional and chiral porphyrins: Model receptors for chiral recognition. Acc. Chem. Res. 1998, 31, 81-89.

12. Kyba, E.B.; Koga, K.; Sousa, L.R.; Siegel, M.G.; Cram, D.J. Chiral recognition in molecular complexing. J. Am. Chem. Soc. 1973, 95, 2692-2693.

13. Lehn, J.M.; Simon, J.; Moradpour, A. Synthesis and properties of chiral macrotricyclic ligands. Complexation and transport of chiral molecular cations and anions. Helv. Chim. Acta 1978, 61, 2407-2418.

14. Noyori, R. Chiral metal complexes as discriminating molecular catalysts. Science 1990, 248, 1194-1199.

15. Groves, J.T.; Ahn, K.H. Characterization of an Oxoruthenium(IV) porphyrin complex. Inorg. Chem. 1987, 26, 3831-3833.

16. Galardon, E.; Le Maux, P.; Bondon, A.; Simonneaux, G. Chiral recognition of amino esters by a ruthenium porphyrin complex: Kinetics of the exchange process determined by ${ }^{1} \mathrm{H}$ NMR. Tetrahedron Asymmetry 1999, 10, 4203-4210. 
17. Morice, C.; Le Maux, P.; Simonneaux, G.; Toupet, L. Chiral recognition of amino esters by ruthenium porphyrin complexes and crystal structure of \{5,10,15,20-tetrakis[o-(3,3,3-trifluoro-2methoxy-2-phenylpropanoylamino)phenyl]porphyrin\}-bis(L-valine methyl ester)ruthenium(II) ( $\alpha, \alpha, \beta, \beta$ isomer). Dalton Trans. 1998, 4165-4171.

18. Nicolas, I.; Chevance, S.; Maux, P.L.; Simonneaux, G. Chiral recognition of amines and amino acid derivatives by optically active ruthenium Halterman porphyrins in organic solvents and water. Tetrahedron Asymmetry 2010, 21, 1788-1792.

19. Eaton, S.S.; Eaton, G.R.; Holm, R.H. Inter- and intramolecular ligand exchange reactions of Ruthenium(II) carbonyl porphine complexes with nitrogen bases. J. Organomet. Chem. 1972, 39, 179-195.

20. Halterman, R.L.; Jan, S.T.; Nimmons, H.L.; Standlee, D.J.; Khan, M.A. Synthesis and catalytic reactivity of $\mathrm{D}_{4}$-Symmetric dinorbornabenzene-derived metallo tetraarylporphyrins. Tetrahedron 1997, 53, 11257-11276.

21. Imai, H.; Munakata, H.; Uemori, Y.; Sakura, N. Chiral Recognition of amino acids and dipeptides by a water-soluble zinc porphyrin. Inorg. Chem. 2004, 43, 1211-1213.

22. Cort, A.D.; De Bernardin, P.; Schiaffino, L. A new water soluble Zn-salophen derivative as a receptor for $\alpha$-aminoacids: Unexpected chiral discrimination. Chirality 2009, 21, 104-109.

23. Le Maux, P.; Bahri, H.; Simonneaux, G. Molecular recognition of racemic phosphines by a chiral ruthenium porphyrin. J. Chem. Soc. Chem. Commun. 1991, 1350-1352.

24. Galardon, E.; Lukas, M.; Le Maux, P.; Simonneaux, G. Synthesis and characterisation of a new chiral Ruthenium picket-fence porphyrin and its use in chiral recognition of racemic lsocyanides. Tetrahedron Lett. 1999, 40, 2753-2756.

25. Ini, S.; Kapon, M.; Cohen, S.; Gross, Z. Self assembly assisted preparation of a homochiral porphyrin. Tetrahedron Asymmetry 1996, 7, 659-662.

26. Knowles, W.S.; Sabacky, M.J. Catalytic asymmetric hydrogenation employing a soluble optically active Rhodium complex. Chem. Commun. 1968, 1445-1446.

27. Horner, L.; Siegel, H.; Buthe, H. Asymmetric catalytic hydrogenation with an optically active phosphinerhodium complex in homogeneous solution. Angew. Chem. Int. Ed. Engl. 1968, 7, 942.

28. Halpern, J.; Trost, B. Asymmetric catalysis. Proc. Natl. Acad. Sci. USA 2004, 101, 5347.

29. Che, C.M.; Lo, V.K.Y.; Zhou, C.Y.; Huang, J.S. Selective functionalisation of saturated C-H bonds with metalloporphyrin catalysts. Chem. Soc. Rev. 2011, 40, 1950-1975.

30. Lu, H.; Zhang, X.P. Catalytic C-H functionalization by metalloporphyrins: Recent developments and future directions. Chem. Soc. Rev. 2011, 40, 1899-1909.

31. Doyle, M.P.; Forbes, D.C. Recent advances in asymmetric catalytic metal carbene transformations. Chem. Rev. 1998, 98, 911-935.

32. Collman, J.P.; Rose, E.; Venburg, G.D. Reactivity of Ruthenium 5,10,15,20-tetramesitylporphyrin towards Diazoesters: Formation of olefins. J. Chem. Soc. Chem. Commun. 1993, 934-935.

33. Galardon, E.; Le Maux, P.; Simonneaux, G. Cyclopropanation of alkenes with ethyl diazoacetate catalysed by Ruthenium porphyrin complexes. J. Chem. Soc. Chem. Commun. 1997, 927-928.

34. Ferrand, Y.; Le Maux, P.; Simonneaux, G. Highly enantioselective synthesis of cyclopropylphosphonates catalyzed by chiral Ruthenium porphyrins. Org. Lett. 2004, 6, 3211-3214. 
35. Frauenkron, M.; Berkessel, A. A novel chiral Ruthenium porphyrin as highly efficient and selective catalyst for asymmetric cyclopropanations. Tetrahedron Lett. 1997, 38, 7175-7176.

36. Lo, W.C.; Che, C.M.; Cheng, K.F.; Mak, T.C.W. Catalytic and asymmetric cyclopropanation of styrenes catalysed by ruthenium porphyrin and porphycene complexes. J. Chem. Soc. Chem. Commun. 1997, 1205-1206.

37. Gross, Z.; Galili, N.; Simkhovich, L. Metalloporphyrin catalyzed asymmetric cyclopropanation of olefins. Tetrahedron Lett. 1999, 40, 1571-1574.

38. Lane, B.S.; Burgess, K. Metal-catalyzed epoxidations of alkenes with hydrogen peroxide. Chem. Rev. 2003, 103, 2457-2474.

39. De Faveri, G.; Ilyashenko, G.; Watkinson, M. Recent advances in catalytic asymmetric epoxidation using the environmentally benign oxidant hydrogen peroxide and its derivatives. Chem. Soc. Rev. 2011, 40, 1722-1760.

40. Arends, I.W.C.E. Metal-catalyzed asymmetric epoxidations of terminal olefins using hydrogen peroxide as the oxidant. Angew. Chem. Int. Ed. 2006, 45, 6250-6252.

41. Ferrand, Y.; Daviaud, R.; Le Maux, P.; Simonneaux, G. Catalytic asymmetric oxidation of sulfide and styrene derivatives using macroporous resins containing chiral metalloporphyrins $(\mathrm{Fe}, \mathrm{Ru})$. Tetrahedron Asymmetry 2006, 17, 952-960.

42. Le Maux, P.; Simonneaux, G. First enantioselective iron-porphyrin-catalyzed sulfide oxidation with aqueous hydrogen peroxide. Chem. Commun. 2011, 47, 6957-6959.

43. Le Maux, P.; Srour, H.; Simonneaux, G. Enantioselective water-soluble iron porphyrin-catalyzed epoxidation with aqueous hydrogen peroxide and hydroxylation with iodobenzene diacetate. Tetrahedron 2012, 68, 5824-5828.

44. Srour, H.; Le Maux, P.; Simonneaux, G. Enantioselective Manganese-porphyrin-catalyzed epoxidation and $\mathrm{C}-\mathrm{H}$ hydroxylation with hydrogen peroxide in water/methanol solutions. Inorg. Chem. 2012, 51, 5850-5856.

45. Nicolas, I.; Le Maux, P.; Simonneaux, G. Synthesis of chiral water-soluble metalloporphyrins (Fe, $\mathrm{Ru}$,): New catalysts for asymmetric carbene transfer in water. Tetrahedron Lett. 2008, 49, 5793-5795.

46. Halterman, R.L.; Jan, S.T. Catalytic asymmetric epoxidation of unfunctionalized alkenes using the first $\mathrm{D}_{4}$-symmetric metallotetraphenylporphyrin. J. Org. Chem. 1991, 56, 5253-5254.

47. Meng, L.; Cheng, Q.; Kim, C.; Gao, W.Y.; Wojtas, L.; Chen, Y.S.; Zaworotko, M.J.; Zhang, X.P.; $\mathrm{Ma}, \mathrm{S}$. Crystal engeenering of a microporous, catalytically active fcu topology MOF using a custom-designed metalloporphyrin linker. Angew. Chem. Int. Ed. 2012, 51, 10082-10085.

(C) 2014 by the authors; licensee MDPI, Basel, Switzerland. This article is an open access article distributed under the terms and conditions of the Creative Commons Attribution license (http://creativecommons.org/licenses/by/3.0/). 